\title{
NOTES
}

\section{Water Proton Relaxation of Casein Micelle}

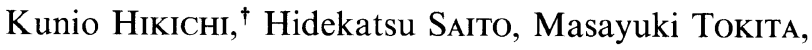 \\ and Ryoya NiKI* \\ Department of Polymer Science, Faculty of Science, and \\ * Institute of Dairy Science, Faculty of Agriculture, \\ Hokkaido University, Sapporo 060, Japan
}

(Received March 18, 1991)

\begin{abstract}
KEY WORDS Casein Micelle / Submicelle / Water Molecules / NMR / Spin-Spin Relaxation Time /
\end{abstract}

Casein is a main component of milk proteins. It forms a protective colloid in milk. The colloidal particle is called a whole casein micelle. The casein micelle is on average $1,400 \AA$ in diameter. ${ }^{1}$ The casein micelle itself is composed of a number of casein submicelles of about $100 \AA$ in diameter, which are connected one another by calcium phosphate. ${ }^{1}$

An important characteristic of casein micelles is their high degree of hydration. They can hold about $4 \mathrm{~g}$ water $/ \mathrm{g}$ casein. The property of water molecules in casein micelle solution is hence one of important subjects to be studied. ${ }^{2}$ In this work, we carried out nuclear magnetic resonance (NMR) experiment to clarify the motional state of water molecules in casein micelle solution. The spin-spin relaxation time $\mathrm{T}_{2}$ of water protons was measured as a function of concentration for casein micelles with different size. The results are discussed in terms of structure of casein micelle.

\section{EXPERIMENTAL}

Skim milk was prepared from fresh milk obtained from the university farm. Three samples of whole casein micelle in different size were prepared by fractionation of skim milk using ultra-centrifugation at different $g$ 's. The samples were finally obtained in a form of pellet. The molecular weights of the three samples were determined by the wavelength dependence of turbidity ${ }^{3}$ and found to be $29.4 \times 10^{9}, 21 \times 10^{9}$, and $0.85 \times 10^{9}$, respectively. A sample of submicelle was also prepared by de-calcifying casein micelle solution. ${ }^{4}$ The molecular weight of submicelle was found to be about $10^{6}$. Artificial milk serum was prepared by the method reported elsewhere. ${ }^{4} \mathrm{H}_{2} \mathrm{O}$ in the serum was partly replaced with $\mathrm{D}_{2} \mathrm{O}$ for field-lock of NMR experiment. The $\mathrm{D}_{2} \mathrm{O} / \mathrm{H}_{2} \mathrm{O}$ ratio was $0.25-0.4$. Casein micelle in the form of pellet was suspended in $2 \mathrm{ml}$ of the serum in a $10 \mathrm{~mm}$ NMR tube. The casein concentration is expressed in weight of dry casein per weight of solution. The NMR measurement was performed on a JEOL JNM-FX60Q spectrometer at a frequency of $60 \mathrm{MHz}$. The spin-spin relaxation time $T_{2}$ of water protons was measured by the Carr-Purcell-Meiboom-Gill method. ${ }^{5}$ The NMR sample tube was not spun during measurements. For all samples the spin-echo decay was a single exponential.

\footnotetext{
† To whom correspondence should be addressed.
} 


\section{RESULTS AND DISCUSSION}

Figure 1 shows the observed relaxation rate (the inverse of $T_{2 \text { obs }}$ ) of water protons at room temperature as a function of casein concentration for four different samples. The relaxation rate $1 / T_{2 \text { obs }}$ increases linearly with the casein concentration. We found that the relaxation rate increases with increasing temperature (the data are not shown here). The results of concentration and temperature dependences of the relaxation rate indicate that water molecules undergo rapid exchange between free and bound states. Figure 1 indicates that the relaxation rate does not depend on the size of whole micelle. There is, however, a remarkable difference between whole casein micelle and submicelle. The slope of the straight line is much smaller for submicelle than for whole micelle.

When water molecules undergo rapid exchange between free and bound states, the observed relaxation rate $1 / T_{2 \mathrm{obs}}$ is given by ${ }^{6}$

$$
\begin{aligned}
1 / T_{2 \mathrm{obs}}= & (1-18 q C / M) / T_{2 \mathrm{f}} \\
& +(18 q C / M) / T_{2 \mathrm{~b}} .
\end{aligned}
$$

Here, $T_{2 \mathrm{f}}$ and $T_{2 \mathrm{~b}}$ are spin-spin relaxation times of water protons in the free and bound states, respectively, $q$ is the number of binding sites per micelle (or submicelle), $C$ is the concentration of casein micelle (submicelle), and $M$ is

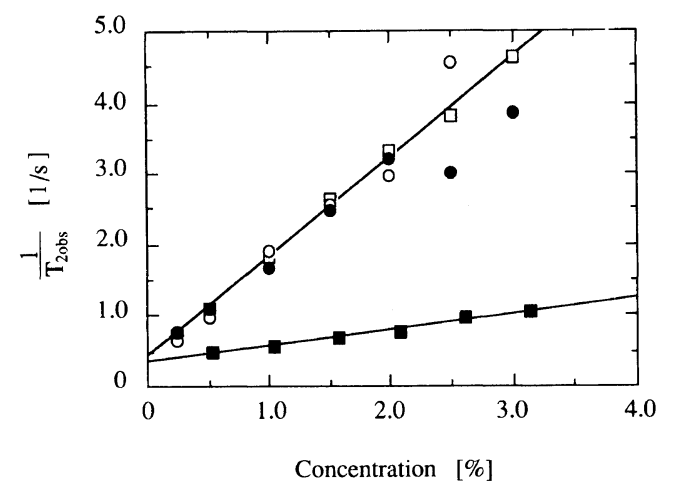

Figure 1. Plots of $1 / T_{2 \text { obs }} v s$. concentration for three different casein micelles $(\boldsymbol{O}, \bigcirc, \square)$ and sub-micelle $(\square)$ at room temperature. the molecular weight of micelle (submicelle). $T_{2 \mathrm{f}}$ and $T_{2 \mathrm{~b}}$ are given by the following equation. ${ }^{7}$

$$
1 / T_{2 \mathrm{f}, \mathrm{b}}=(3 / 2)\left(\gamma^{4} \hbar^{2} \tau_{\mathrm{f}, \mathrm{b}} / r^{6}\right) .
$$

Here, $\gamma$ is the magnetogyric ratio, $\hbar$ the Planck constant divided by $2 \pi, r$ the interproton distance of water molecule, and $\tau_{\mathrm{f}}$ and $\tau_{\mathbf{b}}$ are rotational correlation times in the free and the bound states, respectively.

We examine two models of micellar (submicellar) structure from the view-point of binding of water molecule. The first is that the casein micelle (submicelle) is a rigid sphere of volume $V$ to which water molecule binds. The second is that the micelle (submicelle) is not a single rigid sphere but consists of a large number of mobile sub-units of volume $v$ and molecular weight $m$ to which water molecule binds; these sub-units undergo tumbling motion inside micelle more or less independently.

The slope of the straight line $\mathrm{S}$ of Figure 1 can be calculated under the assumption that the correlation time in the bound state is equal to that of micelle (model 1) or sub-unit (model 2) and obeys the Einstein-Stokes equation. Using the interproton distance $r=1.91 \AA$, the viscosity of water at room temperature of 0.01 poise, and other known universal constants, we get for model 1

$$
S=13 q V / M,
$$

and for model 2

$$
S=13\left(q^{\prime} v / m\right) .
$$

Here, $q^{\prime}$ is the number of binding sites per sub-unit. We neglect the contribution from the free water, because the correlation time in the free state is much smaller than that in the bound state.

The number of binding sites of water molecule per micelle (submicelle) $q$ is reasonably assumed to increase with increasing micelle-size. $V / M$ is the specific volume of micelle (submicelle), and seems independent 
of the micelle-size. Equation 3, thus, indicates that $S$ increases with increasing micelle-size. As seen in Figure 1, $S$ does not, however, depend on the micelle-size. We exclude model 1 and adopt model 2 .

The factor of $v / m$ in eq 4 expresses the specific volume of the sub-unit, and is assumed to be of the order of unity. From the observed values of slope, $q^{\prime}$ is estimated to be 10 for whole casein micelle and 2 for submicelle. These small values of $q^{\prime}$ imply that the sub-unit is small in size. Since $q^{\prime}$ is the number of the "exchangeable" water binding sites, the larger value of $q^{\prime}$ for whole micelle than for submicelle means that the whole micelle has a larger number of exchangeable water molecules than submicelle. This is presumably ascribed to the hydration of calcium phosphates which bind submicelles.

In conclusion, we are led to an idea that casein micelle (submicelle) is composed of a large number of small sub-units which independently undergo tumbling motion inside micelle. This idea is in agreement with the previous model of a loose sponge-like structure of casein micelle. ${ }^{8}$

Acknowledgments. The authors would like to thank Dr. Y. Sano of National Food
Research Institute of Japan for measuring molecular weight of casein micelle. This work was supported by Grants-in-Aid for Scientific Research (No. 0150684 and No. 02660287) from the Ministry of Education, Science, and Culture of Japan.

\section{REFERENCES}

1. H. M. Farrel, in "Fundamentals of Dairy Chemistry," N. P. Wong, R. Jenness, M. Keeney, and E. H. Marth, Ed., Van Nostrand Reinhold, New York, N.Y., 1988, p 461.

2. D. G. Schmidt, in "Developments in Dairy Chemistry-1 Proteins," P. F. Fox, Ed., Elsevier, London, 1986, p 61.

3. Y. Sano and M. Nakagaki, J. Phys. Chem., 87, 1614 (1983).

4. Ryoya Niki, Nippon Nōgeikagaku Kaishi, 61, 1087 (1987).

5. H. Y. Carr and E. M. Purcell, Phys. Rev., 94, 630 (1954); S. Meiboom and D. Gill, Rev. Sci. Instr., 29, 688 (1958).

6. O. Jardetzky and G. C. K. Roberts, "NMR in Molecular Biology," Academic Press, New York, N.Y., 1981, Capter IV.

7. A. Abragam, "The Principles of Nuclear Magnetism," Clarendon Press, Oxford, 1961, Chapter VIII.

8. D. G. Schmidt and T. A. J. Payens, in "Surface and Colloidal Science," Vol. 9, E. Matijevic, Ed., John Wiley \& Son, New York, N.Y., 1976, p 181. 